\title{
Breast cancer: demographic characteristics and clinico-pathological presentation of patients in Iraq
}

N.A.S. Alwan

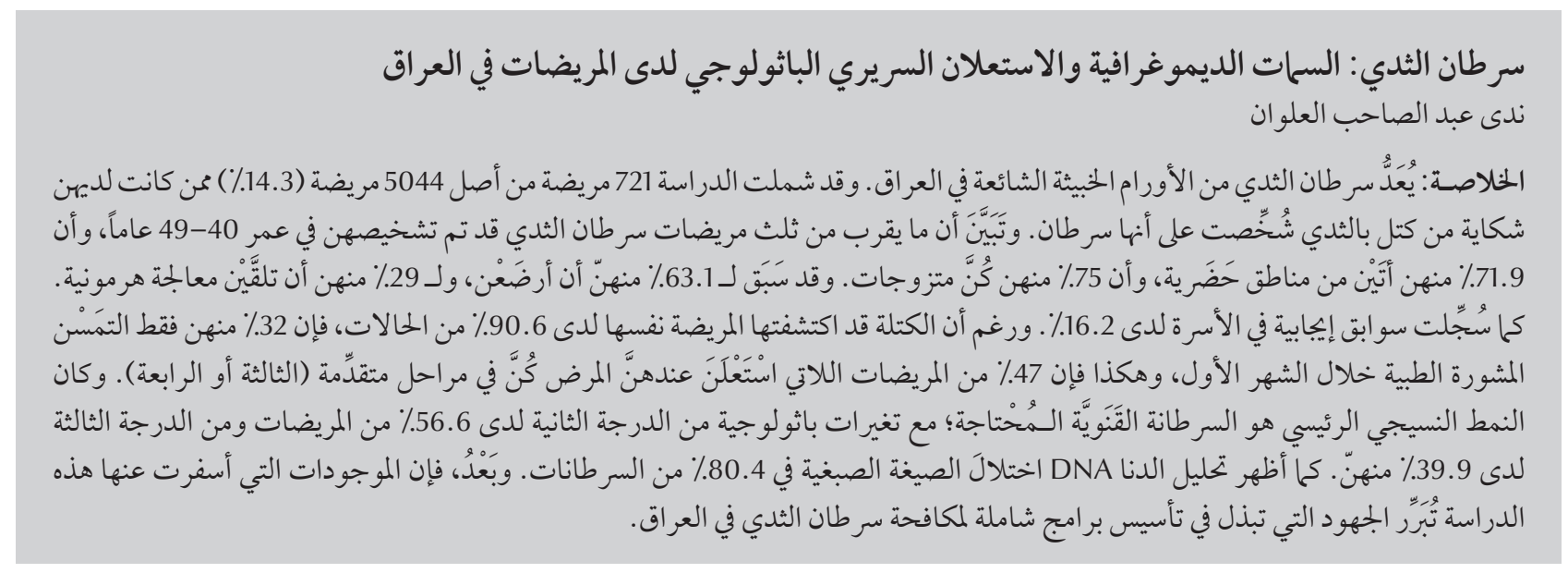

ABSTRACT Breast cancer is the commonest type of malignancy in Iraq. The study was carried out on 721 out of a total of 5044 patients (14.3\%) presenting with palpable breast lumps that were diagnosed as cancer. Approximately one third of the breast cancer patients were diagnosed at age 40-49 years; $71.9 \%$ came from urban areas; and $75 \%$ were married. History of lactation was reported in $63.1 \%$ and hormonal therapy in $29 \%$. Positive family history was recorded in $16.2 \%$. Although the lump was detected by the patient herself in $90.6 \%$ of cases, only $32 \%$ sought medical advice within the first month. Accordingly, $47 \%$ of these patients presented in advanced stages (III and IV). The main histological type was invasive ductal carcinoma, in which pathological changes of grade II and III were observed in 56.6\% and $39.9 \%$ respectively. DNA analysis showed that $80.3 \%$ of the carcinomas were aneuploid. The findings of this study justify increasing efforts for establishing comprehensive breast cancer control programmes in Iraq.

Cancer du sein : caractéristiques démographiques des patientes et présentation clinico-pathologique en Iraq

RÉSUMÉ Le cancer du sein est le type de malignité le plus courant en Iraq. L'étude a été menée sur 721 patientes sur un total de 5044 (14,3\%) se plaignant d'une grosseur au sein palpable qui a été diagnostiquée comme cancer. Environ un tiers des patientes a été diagnostiqué entre 40 et 49 ans ; 71,9\% venaient de zones urbaines; et $75 \%$ étaient mariées. Des antécédents d'allaitement ont été signalés dans 63,1\% des cas et une hormonothérapie dans $29 \%$. Des antécédents familiaux positifs ont été recensés dans 16,2\% des cas. Bien que la grosseur ait été détectée par la patiente elle-même dans 90,6\% des cas, seulement $32 \%$ d'entres elles ont consulté un médecin au cours du premier mois. En conséquence, $47 \%$ de ces patients présentaient un stade avancé (III et IV). Le principal type histologique était celui du carcinome canalaire invasif, dans lequel des changements pathologiques de grade II et III ont été observés dans 56,6 \% et 39,9\% des cas respectivement. L'analyse ADN a révélé que 80,3 \% des carcinomes étaient aneuplö̈des. Les résultats de cette étude justifient un renforcement des efforts visant à mettre en place des programmes complets de lutte contre le cancer du sein en Iraq.

'Breast Cancer Research Unit, Medical College, Baghdad University, Baghdad, Iraq (Correspondence to N.A.S. Alwan: nadalwan@yahoo.com). Received: 16/09/09; accepted: 31/03/10 


\section{Introduction}

Globally, breast cancer is the most common cancer among women, comprising $23 \%$ of the 1.1 million female cancers that are newly diagnosed each year $[1,2]$. It is also the leading cause of cancer-related deaths worldwide, case fatality rates being highest in low resource countries [3]. Approximately 4.4 million women diagnosed with breast cancer in the last 5 years are still alive, making breast cancer the most prevalent cancer worldwide [1].

In Iraq, breast cancer is the commonest type of female malignancy, accounting for approximately one-third of the registered female cancers according to the latest Iraqi Cancer Registry [4]. This shows that the breast is the leading cancer site among the Iraqi population in general, surpassing even bronchogenic cancer.

As proposed by the World Health Organization, early detection and screening, especially when combined with adequate therapy, offer the most immediate hope for a reduction in breast cancer mortality [5]. This was the basis of the Iraqi national programme for early detection of breast cancer, which was initiated in 2001 in an attempt to downstage this disease at the time of presentation. Since then specialized centres and clinics for early detection of breast tumours have been established in the major hospitals in all Iraqi provinces.

This report reviews the main demographic characteristics and clinicopathological presentation in 721 female Iraqi patients who were diagnosed as having breast cancer within the Main Referral Training Centre for Early Detection of Breast Tumours in Baghdad.

\section{Methods}

\section{Case recording and clinical examination}

The present study covered a total of 5054 female patients who visited the
Main Referral Training Centre for Early Detection of Breast Tumours in the Medical City Teaching Hospital in Baghdad during the period February 2004-April 2008 presenting with palpable breast lumps. Patients were clinically interviewed and examined using a triple assessment technique, i.e. clinical breast examination, mammography and/or ultrasonography, and fine needle aspiration cytology (FNAC).

Data routinely recorded on the patient's file sheet questionnaire by the examining physician included age; marital status; residence; history of lactation, contraceptive pills and/ or hormonal therapy; and family history of breast cancer. Data on tumour size and nodal status were obtained by examination of the tissue biopsies. Abdominal ultrasound and chest X-rays were carried out to exclude metastasis, and when indicated a skeletal survey was performed.

\section{Pathological diagnosis}

Patients were scheduled for complete diagnostic work-up, which comprised FNAC, and excisional biopsy and/or mastectomy. Fine needle aspiration was carried out using disposable $10 \mathrm{~mL}$ syringes attached to 22- or 23-gauge needles. The aspirated material was immediately fixed in 99\% ethanol for at least 20 minutes and then stained with Papanicolaou stain. All reports with positive FNAC results were confirmed histopathologically after excision of the lesions. Tissue sections of 721 (14.3\%) formalin-fixed, paraffin-embedded specimens were stained with haematoxylin and eosin and stored for further analysis. Carcinoma type was determined following the WHO classification [6] while the TNM (tumour, node, metastasis) staging system of the American Joint Committee on Cancer (AJCC) was used in recording the clinical stage of the disease [7]. Ductal carcinoma was graded following the recommendations of Scarff, Bloom and Richardson [8].

\section{Assessment of nuclear DNA ploidy content}

The procedure for quantitative cytophotometric DNA analysis and nuclear DNA ploidy assessment was applied on 137 pathological specimens using image cytometry according to the Feulgin technique [9]. Specimens were selected according to the protocol that for each slide, there was an adequate number $(\geq 200)$ of tumour cells and upon admixture with normal lymphocytes which were used as internal controls of the normal diploid (i.e. 2C) DNA content in all specimens. All tumour values were expressed in $\mathrm{C}$ units in relation to the corresponding internal controls. The DNA histogram patterns were classified according to the criteria described by Auer et al. [9] into type I (diploid), type II (tetraploid), or types III and IV (aneuploid).

\section{Determination of estrogen and progesterone receptor content}

For the determination of estrogen receptor (ER) and progesterone receptor (PR) contents, a semiquantitative immunocytochemical assay (ERICA/ PRICA) was used on 125 cases using Abbott reagents (the number was restricted according to the availability of fine needle aspirates having an adequate number of tumour cells). Briefly, the method included incubation of breast cancer cells with the primary antibody (an IgG fraction of monoclonal rat antibody to human ER or PR) and a bridging goat antibody. The reaction of peroxidase with hydrogen peroxide yielded an insoluble reddish brown product which was visualized by light microscopy. The receptor cells, located inside the nuclei of hormone receptor positive tumour cells, stained reddish brown when positive and light grey when negative. During analysis the staining intensity (weak $=1$; moderate $=2$; intense $=3$ ) and the percentage of positively stained cells were combined as primary parameters using a threshold 
value of 20 , e.g. moderate staining (=2) would need $\geq 10 \%$ of cells showing a positive staining reaction $(2 \times 10=20)$ [10].

\section{Her-2/neu immunohistochemical staining}

Sections $5 \mu$ thick were made from formalin fixed-paraffin embedded tissue blocks belonging to 192 patients were stained immunohistochemically through streptavidin biotin peroxidase complex technique using anti-Her-2/ neu monoclonal antibody (rabbit antihuman Her-2 protein/Dakocytomation). The number tested was limited by the availability of specimens having an adequate number of tumour cells and the availability of the immunocytochemical kits. The intensity of the cancerous cell membrane staining of Her-2 neu protein expression was graded through a score of 0 to $3+$ recording the intensity of the staining reaction in more than $10 \%$ of tumour cells; whereby $2+$ over expression was regarded as weak positive and $3+$ as strong positive.

\section{Results}

Pathological diagnosis showed cellular changes consistent with mammary carcinoma in smears belonging to 721 (14.3\%) of the 5054 patients presenting with breast lumps. The peak frequency of breast cancer increased with age until menopause, thereafter it started to decline. More than half the patients (54.1\%) were in the premenopausal age, and $22.2 \%$ were under 40 years (Table 1).

Family history of breast cancer was reported in 177 patients (16.2\%); 35 of these (19.8\%) had an affected first degree relative. Although the malignant lump was detected by the patient herself in 653 cases (90.6\%), only $32.5 \%$ of these sought medical advice within the first month after detection while $16.2 \%$ did so after 1 year.

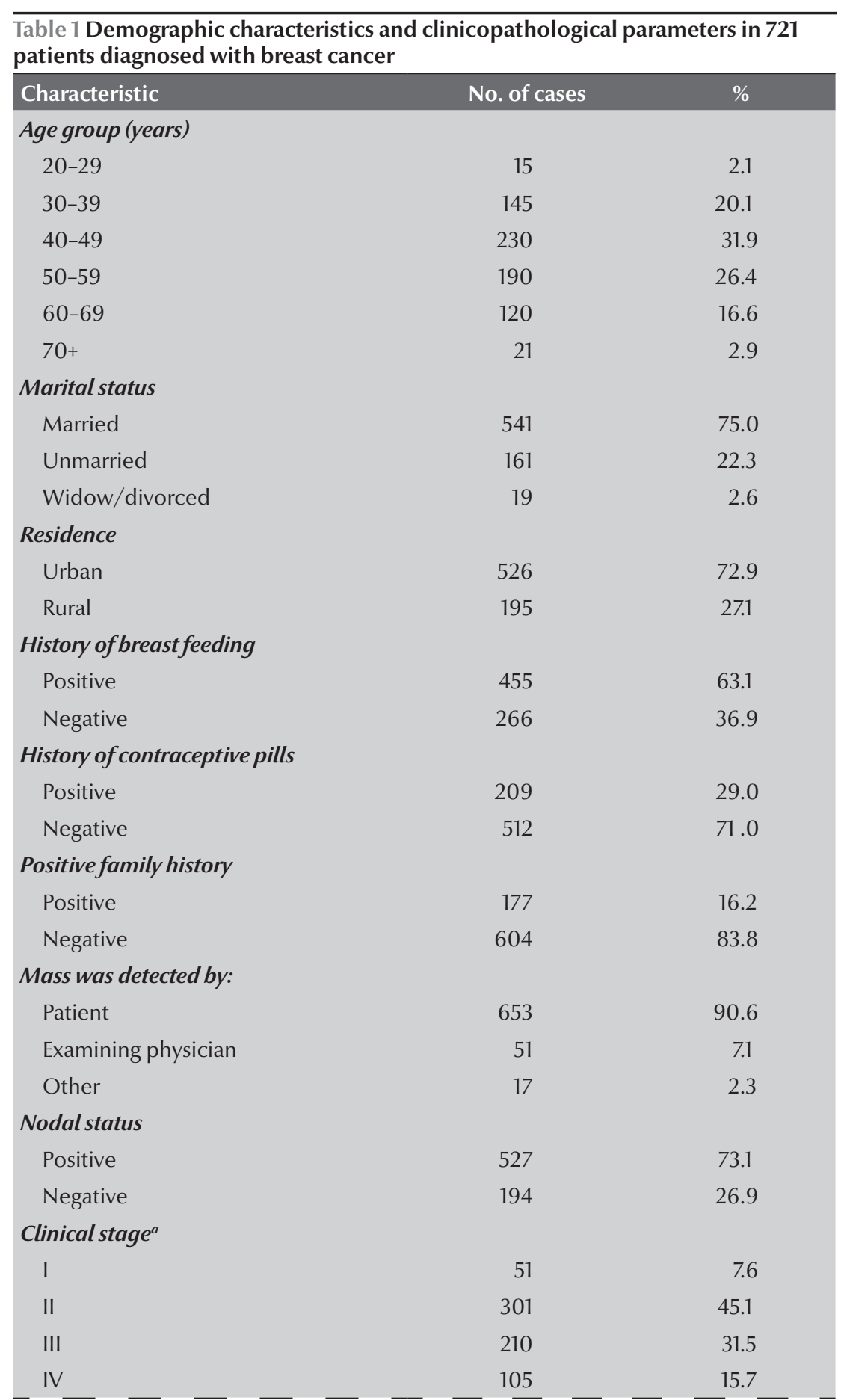

Following the WHO classification, the most common histological type determined microscopically was invasive ductal carcinoma (655 patients: $90.8 \%)$. At the time of initial diagnosis 527 patients (73.1\%) had axillary nodal involvement. According to the AJCC system, the frequencies were $7.6 \%, 45.1 \%, 31.5 \%$ and $15.7 \%$ for stages I, II, III and IV respectively.
Scarff-Bloom-Richardson classification showed that $3.5 \%, 56.6 \%$ and $39.9 \%$ of ductal carcinomas were graded as I, II and III respectively.

Cytophotometric DNA analysis showed that $80.3 \%$ (110 out of 137 ) of examined mammary carcinomas was aneuploid (Table 2). According to the histogram classification of Auer et al., $11.6 \%$ were diploid, $8.1 \%$ were 


\begin{tabular}{|c|c|c|}
\hline Characteristic & No. of cases & $\%$ \\
\hline \multicolumn{3}{|l|}{ Tumourgrade } \\
\hline I & 25 & 3.5 \\
\hline II & 408 & 56.6 \\
\hline III & 288 & 39.9 \\
\hline \multicolumn{3}{|c|}{ Nuclear DNA ploidy tumour content ${ }^{b}$} \\
\hline Euploid & 27 & 19.7 \\
\hline Aneuploid & 110 & 80.3 \\
\hline \multicolumn{3}{|c|}{ Hormone receptor tumour content $t^{c}$} \\
\hline $\mathrm{ER}+$ & 166 & 65.1 \\
\hline $\mathrm{PR}+$ & 115 & 45.1 \\
\hline \multicolumn{3}{|c|}{ Her-2/neu tumour expression ${ }^{d}$} \\
\hline Positive & 89 & 46.4 \\
\hline Negative & 103 & 53.6 \\
\hline Total & 721 & \\
\hline
\end{tabular}

a Total number of patients classified according to the American Joint Committee on Cancer Staging system $=667$. ${ }^{b}$ Total number of specimens analysed for nuclear DNA ploidy tumour content $=137$.

'Total number of specimens analysed for HR content $=255$.

${ }^{d}$ Total number of specimens analysed for Her-2 neu expression $=192$. tetraploid, $49.6 \%$ were aneuploid and $30.7 \%$ were highly aneuploid. Among the poorly differentiated carcinomas (Grade III) 84.9\%were aneuploid ( $P$ $<0.005)$

ERandPR positive tumour contents were demonstrated in $166(65.1 \%)$ and 115 (45.1\%) respectively of 255 specimens examined; the ER+/PR+ phenotype being recoded in $40.8 \%$ of the studied cases, while the ER-/PR-variant was displayed in 30.6\% (Table 3). Approximately $72 \%$ of postmenopausal breast cancers were ER+ $(P<0.05)$. Poorly differentiated carcinomas exhibited ER+ and PR+ phenotypes in $41.1 \%$ and $29.6 \%$ respectively $(P<0.05)$.

Immunohistochemical staining for Her-2/neu protein over-expression showed a positive reaction in $46.4 \%$ ( 89 out of 192 examined tissue blocks); $92 \%$ of grade I mammary ductal carcinomas showed negative Her-2/neu expression, whereas $61.7 \%$ and $73 \%$ of grade II and grade III carcinomas respectively were positive.

\section{Discussion}

According to WHO mortality estimates, cancer is the fourth ranked cause of death in the Eastern Mediterranean Region (EMR), after cardiovascular diseases, infectious/parasitic diseases and injuries [11]. The largest increase in cancer incidence among the WHO regions in the next 15 years is likely to be

\begin{tabular}{lcc}
\hline $\begin{array}{l}\text { Table } 2 \text { DNA histogram types and nuclear DNA ploidy content distribution in } \\
\text { pathological specimens belonging to }\end{array} \mathbf{1 3 7}$ breast cancer patients \\
\hline DNA histogram type & No. of cases & $\%$ \\
I (diploid) & 16 & 11.6 \\
II (tetraploid) & 11 & 8.1 \\
III (aneuploid) & 68 & 49.6 \\
IV (aneuploid) & 42 & 30.7 \\
Total & 137 & \\
\hline
\end{tabular}

in the EMR [12], where breast cancer is recorded as the commonest type of female malignancy in almost all national cancer registries.

In Iraq, in addition to being the most important cancer, there are other features that justify increasing efforts for breast cancer control including the tendency for this disease to affect younger women, the obvious rise in incidence rates and the prevalence of advanced stages at presentation associated with more aggressive tumour behaviour resulting in greater fatality rates.

In this study, breast cancer was diagnosed in $14.3 \%$ of patients presenting with apparent breast lumps. Approximately one third of the patients were diagnosed in their forties, where the peak frequency occurred, while an obvious decline was displayed after the age of 60 years. This continuing trend for this disease to affect younger generations has been comprehensively illustrated in the Iraqi Cancer Registry [4] and other documented reports from neighbouring countries. $[13,14] \mathrm{WHO}$ estimates revealed that approximately half of the cancers in the EMR occur before the age of 55 and that the age standardized incidence rates of all cancers in this region is expected to double as risk factor exposure increases [11]. This picture differs from that displayed in reports from western and developed countries; where the peak incidence rates project decades later [15]. In a WHO collaborative project it has been proposed that the younger age distribution in the Arab population could be a reflection of the younger demographic profile [16].

The relatively high frequency of patients with positive family history observed in this study compared to others $[17,18]$ could be attributed to the customary consanguineous marriages which are known to be common throughout the region [19].

Although $90.6 \%$ of the patients detected the lumps by themselves, regrettably, only $32 \%$ sought medical advice 


\begin{tabular}{lcc}
\hline \multicolumn{3}{l}{ Table 3 Distribution of hormone receptor (ER and PR) phenotypes in pathological } \\
specimens belonging to 255 breast cancer patients \\
\hline Hormone receptor phenotype & No. of cases & $\%$ \\
ER+/PR+ & 104 & 40.8 \\
ER+/PR- & 62 & 24.3 \\
ER-/PR+ & 11 & 4.3 \\
ER-/PR- & 78 & 30.6 \\
Total & 255 & 100.0 \\
\hline
\end{tabular}

within the first month after detection. This finding was illustrated by the fact that $73 \%$ of the studied group had positive axillary nodal metastasis at the time of diagnosis; thus placing $47.2 \%$ within stages III and IV. High proportions of advanced stages at presentation have been documented in surveys from other developing countries $[14,20]$. The IARC Working Group indicated that survival from this cancer in middle- and low-income countries remains poor, mainly because of the late presentation [21].

These observations obviously reflect the poor health education of the general population and their ignorance regarding the significance of clinical breast examination, breast self examination and early medical consultation. In a critical evaluation of the role of early detection and screening of breast cancer in developing countries, the late stage at presentation has been attributed to be a reflection of the cultural norms which downplay women's health problems [16]. It is believed that the improved survival rates in many cancers in the United States of America and Europe are more related to the earlier stage at presentation rather than to improved treatment [22].

Pathologically, in accordance with the results of other cancer registries [15], the leading tumour type was infiltrative ductal carcinomas. A direct relationship was noted between the pathological nodal status and the histological grade; where 17 out of 25 patients (68\%) diagnosed as having well differentiated carcinomas
(Grade I) in this study presented with negative axillary nodes while all patients with poorly differentiated breast carcinomas (Grade III) had nodal metastasis at the time of diagnosis, thus confirming that the nodal status might be predicted, at least from the statistical point of view, from the histological grade of the tumour [23].

At the molecular level, it has been documented that complex genomic alterations, reflected by altered DNA content, are involved in tumour development and progression. In this respect, nuclear DNA ploidy content of breast cancer cells has been used to predict the outcome of the disease. In general, patients with genetically stable breast cancer exhibiting DNA diploid or tetraploid profiles have a better prognosis and more favourable clinical course than those with genetically unstable aneuploid cancer [24]. The frequency of aneuploid mammary carcinomas in this study (80.3\%) was considerably higher than the range reported by other investigators from more-developed countries [25]. To explain the observed differences in ploidy pattern distribution, one should consider the possible variations in the biological behaviour of the tumour and the differences in demographic characteristics and clinico-pathological presentations of the studied populations. However, earlier reports documented that DNA ploidy content generally remains stable during cancer progression $[9,24]$; thus providing additional significant prognostic information superior to that displayed by other clinical and histomorphological variables.
No significant correlation was demonstrated regarding the association of aneuploid mammary carcinoma with the age of the patient, nor with the stage of disease at presentation. Nevertheless, a significant association with the grade of mammary carcinoma was clearly displayed: $84.9 \%$ of poorly differentiated carcinomas were aneuploid.

The lower frequencies of hormone receptor (HR) positive mammary carcinomas among Iraqi patients that were reported in this study and others $[26,27]$ compared to those observed more-developed countries [28] are probably expected in a population of predominantly middle-aged patients harbouring considerable rates of poorly-differentiated carcinomas. In general, there is a more uniform loss of these receptor contents as the tumour becomes more anaplastic, indicating that hormone receptor status could represent one aspect of tumour cell differentiation. Our results showed a direct significant association between menopausal status, grade of carcinoma and hormonal receptor positive contents.

Following the same rationale, a relatively high rate of positive Her-2/neu protein expression was demonstrated in this study, similar to findings previously reported from Arab countries [29], and this correlated significantly with the histological tumour grade.

Thus, the coexistence of HER-2/ neu over-expression, hormone receptornegative and nuclear aneuploid tumour contents in the examined tissue could serve as a strong prognostic indicator for the progress of the disease process.

In conclusion, the expanding burden of cancer in the EMR in general, and Iraq in particular, justifies the demand for establishing comprehensive national cancer control programmes. Early detection of breast cancer, as a major approach to controlling the disease, could be achieved by raising the awareness of the general population 
about its symptoms and signs, educating health personnel, and ensuring the provision of readily accessible diagnostic services. The Breast Health Global initiative provided guideline implementation for breast health care in low- and middle-income countries [3]. Pilot projects from Egypt and South Asia have demonstrated provisional evidence for the value of these programmes $[30,31]$.

The illustrated high frequencies of poorly differentiated, aneuploid mammary carcinomas and ER-/PR- phenotypes, as objective biological markers of tumour aggressiveness, emphasize the urgentneed forinitiating comprehensive clinical, interventional and molecular research studies [32]. By combining clinical, pathological, hormonal and morphological determinants, the prognosis of breast cancer patients might become more predictable; thus permitting judicious selection of the most effective therapeutic protocols.

\section{References}

1. Parkin DM et al. Global cancer statistics 2002, CA: A Cancer Journal for Clinicians, 2005, 55:74-108.

2. Parkin DM, Fernandez LM. Use of statistics to assess the global burden of breast cancer. Breast, 2006, 12(1 Suppl.):S70-S80.

3. Anderson $\mathrm{BO}$ et al. Guideline implementation for breast healthcare in low-income and middle-income countries. Overview of the Breast Health Global Initiative Global Summit, 2007. Cancer, 2008, 113(8 Suppl.):2221-2243.

4. Iraqi Cancer Board. Results of the Iraqi Cancer Registry 2004. Baghdad, Iraqi Cancer Registry Center, Ministry of Health, 2007.

5. National Cancer Control Programs. Policies and managerial guidelines, 2nd. ed. Geneva, World Health Organization, 2002.

6. Edge SB et al. AJCC Cancer Staging Manual, 7th ed. New York, Springer-Verlag, 2010.

7. American Joint Committee on Cancer. The breast. In: AJCC cancer staging manual, 6th ed. New York, Springer, 2002:171180.

8. Rosai J, ed. Rosai and Ackerman's surgical pathology: breast, 9th ed, vol. II. St. Louis, Mosby, 2004:1763-1839.

9. Auer GU. DNA and prognosis in breast cancer [thesis]. Stockholm, Karolinska Institute, 1986.

10. Marrazo A et al. Immunocytochemical determination of oestrogen and progesterone receptors on 219 FNA of breast cancer. Anticancer Research, 1995; 15: 521-526.

11. Revised global burden of disease (GBD), WHO 2002 estimates. Geneva, World Health Organization, 2003 (http:// www.who.int/healthinfo/bodgbd2002revised/en/index. html, accessed 1 September 2009).

12. Rastogi T, Hildesheim A, Sinha R. Opportunities for cancer epidemiology in developing countries. Nature Reviews Cancer, 2004, 4(11):909-17.

13. Fakhro A et al. Breast cancer: patient characteristics and survival analysis at Salmaniya Medical Complex, Bahrain. Eastern Mediterranean Health Journal, 1999, 5(3):430-439.

14. Omar S et al. Breast cancer in Egypt: A review of disease presentation and detection strategies. Eastern Mediterranean Health Journal, 2003, 9(3):448-461.

15. Rennert G. Breast cancer. In: Freedman L et al. Cancer incidence in four member counties (Cyprus, Egypt, Israel and Jordan) of the Middle East Cancer Consortium (MECC) compared to SEER. Bethesda, Maryland, NCI (NIH Pub. No. 06-5873), 2006:73-81.

16. Miller AB. Mammography; a critical evaluation of its role in breast cancer screening, especially in developing countries. Journal of Public Health Policy, 1989, 10:486-497.
17. Tada T, Kasumi F. Characteristics of familial breast cancer. Nippon-Rinsho, 2000, 7(58):1405-1408.

18. Anynawu S. Breast cancer in eastern Nigeria: a ten year review. West African Journal of Medicine, 2000, 19(2):120-125.

19. Alwan A et al. Community control of genetic and congenital disorders. Cairo, World Health Organization, 1997 (Technical Publications Series 24:63-79).

20. ISA Hospital Cancer Registry, Tunisia. Registre de l'institut Salah Azaiz 1994-1999. Tunis, Ministère de la Santé, Institut Salah Azaiz, 2000.

21. IARC Working Group on the Evaluation of Cancer-Preventive Strategies. Breast cancer screening. Lyon, World Health Organization, 2002.

22. Etzioni $\mathrm{R}$ et al. The case for early detection. Nature Reviews Cancer, 2003, 3(4):243.

23. Harris GC, Pinder SE, Elson CW. The role of the pathologist in assessing prognostic factors for breast cancer. In: Walker RA. Prognostic and predictive factors in breast cancer. London, Martin Dunitz Group, 2003:7-22.

24. Fallenius AG, Auer GU, Carstensen JM. Prognostic significance of DNA measurements on 409 consecutive breast cancer patients. Cancer , 1988, 62:331-341.

25. Kronenwett U, Ploner A, Zetterberg A et al. Genomic instability and prognosis in breast carcinomas. Cancer Epidemiology Biomarkers \& Prevention, AACR, 2006, 15:6030-6035.

26. Al-Alwan N. DNA proliferative index as a marker in Iraqi aneuploid mammary carcinoma. Eastern Mediterranean Health Journal, 2000, 6(5/6):1062-1072.

27. Al-Alwan N, Al Kubaisi W, Al Rawaq K. Assessment of response to Tamoxifen in Iraqi patients with advanced Breast Cancer. Eastern Mediterranean Health Journal, 2000, 6(3/4):475-482.

28. Elledge RM and Allred DC. Clinical aspects of estrogen and progesterone receptors. In: Harris JR et al., editors. Diseases of the breast. Philadelphia, Lippincott Williams \& Wilkins, 2004:603-17.

29. Khorshid OM et al. Prognostic significance of HER2/neu oncogene in breast cancer patients. Cairo, National Cancer Institute, Scientific Annual Report, 2002.

30. Boulos $\mathrm{S}$ et al. Breast screening in the emerging world: high prevalence of breast cancer in Cairo. The Breast, 2005, 14:340346.

31. Devi B, Tang TS, Corbex M. Reducing by half the percentage of late-stage presentation for breast and cervix cancer over 4 years: a pilot study of clinical downstaging in Sarawak, Malaysia. Annals of Oncology, 2007, 18(7):1172-1176.

32. Mellstedt H. Cancer initiatives in developing countries. Annals of Oncology, 2006, 17(Suppl. 8):viii24-viii31. 